\title{
A REMARK ON ZETA FUNCTIONS
}

\section{H. KOBER}

1. Let $s=\sigma+i t, \zeta(s)=\sum_{1}^{\infty} n^{-\infty}(\sigma>1), \omega(x)=\sum_{n=1}^{\infty} e^{-n^{2} \pi x}(x>0)$,

$$
\xi(s)=\pi^{-8 / 2} \Gamma(s / 2) \zeta(s)=\int_{0}^{\infty} \omega(x) x^{s / 2-1} d x \quad(\sigma>1) .
$$

Then $s(s-1) \xi(s)$ is well known to be an entire function, and its zeros are identical with the nontrivial zeros of $\zeta(s)$, i.e. with those lying in the strip $0<\sigma<1$. Furthermore let

$w=u+i v, w \neq 0 ;|\arg w|,|\arg 1 / w|,|\arg (x+w)| \leqq \pi / 2 ; s \neq 0,1$, be fixed;

$$
F_{s}(w)=\int_{0}^{\infty} \omega(x+w)(x+w)^{s / 2-1} d x-\frac{w^{(8-1) / 2}}{1-s} .
$$

Then $F_{s}(w)$ is an analytic function of $w$ for $u>0$, since

$$
|\omega(x+w)| \leqq x^{-1 / 2} e^{1-x} \quad(x>0) ;
$$

its limit function $F_{s}(i v)$ exists for any $v \gtrless 0$ by the Lebesgue convergence theorem. Now we can deduce that

$$
F_{s}(w)+F_{1-s}(1 / w)=\xi(s) \quad(u \geqq 0, w \neq 0) .
$$

For $v=0$ this reduces to the, possibly known, equation ${ }^{1}$

$$
\xi(s)=\int_{u}^{\infty} \omega(x) x^{s / 2-1} d x+\int_{1 / u}^{\infty} \omega(x) x^{-(1+o) / 2} d x-\frac{u^{(s-1) / 2}}{1-s}-\frac{u^{s / 2}}{s} .
$$

Hence (1.3) hold by analytic continuation. Clearly $F_{s}(w) \rightarrow \xi(s)(w \rightarrow 0$; $0<\sigma<1)$.

Again (1.3) takes simple forms for $w=i$ and $w=2 i$ :

$$
\begin{aligned}
\xi(s)= & \int_{0}^{\infty} \lambda(x)(x+i)^{s / 2-1} d x \\
& +\int_{0}^{\infty} \lambda(x)(x-i)^{-(o+1) / 2} d x-\frac{e^{i \pi(s-1) / 4}}{1-s}-\frac{e^{i \pi s / 4}}{s}
\end{aligned}
$$

Received by the editors June 2, 1952 and, in revised form, November 7, 1952.

${ }^{1}$ For $u=1$ this is the classical equation due to Riemann. E.g. E. Landau, Handbuch der Lehre von der Verteilung der Primsahlen, Leipzig and Berlin, 1909, \$70; by a similar argument (1.3a) is deduced. 


$$
\begin{aligned}
\xi(s)= & \int_{0}^{\infty} \omega(x)(x+2 i)^{s / 2-1} d x \\
& +\int_{0}^{\infty}\{i \omega(x)+(1-i) \omega(4 x)\}\left(x-\frac{i}{2}\right)^{-(s+1) / 2} d x \\
& -\frac{\left(2 e^{i \pi / 2}\right)^{(8-1) / 2}}{1-s}-\frac{\left(2 e^{i \pi / 2}\right)^{\diamond / 2}}{s}
\end{aligned}
$$

where $\lambda(x)=\sum_{1}^{\infty}(-1)^{n} e^{-n^{2} \pi x}$. By the formula $|\Gamma(s)| e^{\pi t / 2} t^{1 / 2-\sigma} \rightarrow$ constant $(0<t \rightarrow \infty)$ the Lindelöf hypothesis ${ }^{2}$ is equivalent to the statement

$$
\begin{aligned}
\Re\left\{\int_{0}^{\infty} \lambda(x)(x+i)^{-3 / 4+i t / 2} d x\right\}= & O\left(t^{-1 / 4+\epsilon} e^{-\pi t / 4}\right) \\
& (\epsilon>0 \text { fixed; } 0<t \rightarrow \infty) .
\end{aligned}
$$

2. The following theorems, easily derived from (1.3), give criteria for the nontrivial zeros of $\zeta(s)$ :

THEOREM 1. A given point s is a nontrivial zero of $\zeta(s)$ if and only if, for $w \neq 0$ with $u \geqq 0, F_{s}(w)$ satisfies the functional equation

$$
F_{s}(w)=-F_{1-s}(1 / w) \text {. }
$$

Theorem 2. Let $0<\sigma<1$. Then $s$ is a zero of $\zeta(s)$ if and only if

$$
\begin{aligned}
& w^{(8-1) / 2} \int_{0}^{\infty}(x+w)^{-(8+1) / 2}\left\{\frac{1}{2}(x+w)^{-1 / 2}-\omega(x+w)\right\} d x \\
& \rightarrow \frac{1}{s-1} \quad(u \geqq 0 ;|w| \rightarrow 0),
\end{aligned}
$$

or, for any fixed $a>0$ (for instance, for $a=1$ ),

$$
\int_{0}^{a} x^{s / 2-1}\left\{\frac{1}{2} x^{-1 / 2}-\rho^{1 / 2} \omega(x \rho)\right\} d x \rightarrow \frac{a^{(\rho-1) / 2}}{s-1} \quad(0<\rho \rightarrow \infty) .
$$

If $s$ is not a zero, the moduli of the terms on the left of (i) and (ii) tend to infinity.

REMARK. For fixed $s \quad(0<\sigma<1), \quad F_{s}(w) \sim w^{(0-1) / 2}(s-1)^{-1} \rightarrow 0$ $(|w| \rightarrow \infty) ; F_{s}(w)$ is bounded and uniformly continuous $(u \geqq 0)$; $F_{s}(w)=(2 \pi)^{-1} \int_{-\infty}^{\infty} d \alpha F_{s}(i \alpha)(w-i \alpha)^{-1} \quad(u>0) ; \quad$ and $^{3} \quad \xi(s)=i / \pi \quad$ PV.

2 The assertion, still unproved, that $\zeta(1 / 2+i t)=O\left(t^{t}\right)(\epsilon>0 ; 0<t \rightarrow \infty)$. This is known to be equivalent to $\int_{0}^{\infty} x^{-3 / 4+i / / 2} \lambda(x) d x=O\left(t^{-1 / 4+e} e^{-\pi t / 4}\right)$.

${ }^{3} \mathrm{PV} . \int_{-\infty}^{\infty}=\lim _{\epsilon \rightarrow 0}\left(\int_{-\infty}^{-i}+\int_{\epsilon}^{\infty}\right)$ is the "principal value" of the integral. The above representations of $F_{s}(w)$ and $\xi(s)$ by integrals follow from the theory of the HilleTamarkin class $\mathfrak{W}_{p}$; see Fund. Math. vol. 25 (1935) pp. 329-352. 
$\int_{-\infty}^{\infty} d \alpha F_{a}(i \alpha) / \alpha$. Beyond the line $u=0$ the function $F_{s}(w)$ can not be continued analytically.

3. In a recent paper T. M. Apostol' has investigated the functional equation of the generalized zeta function $\phi(s, a, b)=\sum_{0}^{\infty} e^{2 \times i n b}(n+a)^{-s}$, due to Lerch, for the case $b \rightarrow 1(0<a \leqq 1,0<b<1)$. The problem can be considerably simplified and, incidentally, generalized. Replace $\phi(s, a, b)$ by $\zeta(s, a, b)$ and introduce $Z_{1}(s, a, b), Z_{2}(s, a, b)$, where $a$ and $b$ are any real numbers,

$$
\begin{aligned}
\zeta(s, a, b) & =\sum_{n>-a} e^{2 \pi i n b}(n+a)^{-s} \\
Z_{1}(s, a, b) & =\sum_{n=-\infty, n+a \neq 0}^{\infty} \frac{e^{2 \pi i n b}}{|n+a|^{*}}, \\
Z_{2}(s, a, b) & =\sum_{n=-\infty, n+a \neq 0}^{\infty} \frac{e^{2 \pi i n b}(n+a)}{|n+a|^{\circ+1}} \quad(\sigma<1) .
\end{aligned}
$$

Obviously

$$
\begin{gathered}
2 \zeta(s, a, b)=Z_{1}(s, a, b)+Z_{2}(s, a, b) ; \\
2 \zeta(s,-a,-b)=Z_{1}(s, a, b)-Z_{2}(s, a, b),
\end{gathered}
$$

and we obtain the functional equations

$$
\begin{aligned}
& e^{2 \pi i a b} \chi(s+k-1) Z_{k}(s, a, b)=i^{k-1} \chi(k-s) Z_{k}(1-s, b,-a) \\
& (k=1,2) \text {, } \\
& \frac{(2 \pi)^{\bullet}}{\Gamma(s)} e^{2 \pi i a b} \zeta(1-s, a, b) \\
& =e^{\pi i \theta / 2} \zeta(s, b,-a)+e^{-\pi i s / 2} \zeta(s,-b, a),
\end{aligned}
$$

where $\chi(s)=\pi^{-s / 2} \Gamma(s / 2) ; a, b$ real. The equation (3.2), known in special cases, ${ }^{5}$ is deduced from well known formulae on theta series, ${ }^{6}$ by the classical method; while, by (3.1), (3.3) is a corollary of it.

\section{Birminghax, ENGLAND}

- Remark on the Hurwits seta function, Proceedings of the American Mathematical Society vol. 2 (1951) pp. 690-693.

E.g. H. Kober, J. Reine Angew. Math. vol. 174 (1936) pp. 206-225, \$4. Again the equation (3.2) for $Z_{k}(s, a, b)$ is deduced by Apostol in the special case $0<a<1$, Pacific Journal of Mathematics vol. 1 (1951) pp. 161-167. For his function $\Lambda(x, a, s)$, defined for $0<a<1$ and treated by the classical method (see pp. 161-163), reduces to $Z_{1}(s, a, x)$, etc. as is easily shown.

- I.e. $\theta(x, a, b)=e^{-8 \pi i a b} x^{-1 / 2} \theta\left(x^{-1}, b,-a\right)$ and the formula gained from this by differentiation with respect to $a$; where $\theta(x, a, b)=\sum_{-\infty}^{-\infty} \exp \left\{-\pi x(n+a)^{2}+2 \pi i n b\right\}$ $=\theta(x,-a,-b)$. 